\title{
Vaccination against COVID-19: insight from arterial and venous thrombosis occurrence using data from VigiBase
}

\begin{abstract}
To the Editor:
Copyright @The authors 2021.

This version is distributed under the terms of the Creative Commons Attribution NonCommercial Licence 4.0. For commercial reproduction rights and permissions contact permissions@ersnet.org

This article has an editorial commentary: https://doi.org/10. 1183/13993003.01111-2021

Received: 31 March 2021 Accepted: 13 April 2021

\section{○@®@}

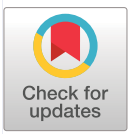

Coronavirus disease 2019 (COVID-19) is associated with a prothrombotic phenotype characterised by coagulopathy and endothelial dysfunction [1-4]. Following some cases of thrombosis after vaccination, the Oxford-AstraZeneca COVID-19 vaccine (AZD1222) was temporarily suspended by some European countries. The European Medicines Agency concluded that the benefits of the vaccine in combating the COVID-19 outbreak continue to outweigh the risk of side-effects. On 19 March, 2021, Germany reported 13 cases of sinus or cerebral vein thrombosis, with more than 1.6 million AstraZeneca COVID-19 vaccine doses administered. Some of these patients also had a heparin-induced thrombocytopenia (HIT)-like syndrome, which suggests an immunological event as one of the potential origins of thrombosis.

Here, we provide a descriptive analysis of the anti-SARS-CoV-2 vaccination thrombotic risk reported to the World Health Organization (WHO) Global Database for Individual Case Safety Reports (VigiBase). VigiBase is a databank developed and maintained by the Uppsala Monitoring Centre, Sweden. It is the world's largest pharmacovigilance database, with submissions from member states since the establishment of the WHO Program for International Drug Monitoring in 1968. Vigibase has been largely used in the past years to detect significant signals for adverse drug reactions [5]. Some adverse drug reactions to vaccines may be identified only after their commercialisation, in particular when the events are very rare or have a delayed time to onset. Therefore, the safety monitoring of vaccines continues in post-marketing surveillance. For example, during the mass vaccination campaign in 2009 for H1N1, several cases of narcolepsy were reported during the post-marketing period [6].

In this context, our study aimed to assess clinical features of arterial and venous thrombosis after injection of three anti-COVID-19 vaccines (Comirnaty ${ }^{\circledR}$ from Pfizer-BioNtech; Moderna COVID-19 vaccine, and AZD1222 from Oxford-AstraZeneca) [7-9] until 16 March, 2021. Between 13 December, 2020 and 16 March, 2021 (94 days), 361734967 people received a vaccination according to the international COVID-19 vaccination dataset [10] and 2161 thrombotic events were reported in Vigibase by 16 March, 2021. Spontaneous reports of thrombotic events are shared in 1197 persons for Comirnaty, 325 for the Moderna COVID-19 vaccine and 639 for AZD1222 (table 1). With these data, we were able to evaluate a reporting rate for venous (VTE) and arterial (ATE) thrombotic events cases during the time period (94 days) among the total number of people vaccinated using the following formula: number of thrombotic cases in the given time period divided by the total numbers of vaccinated person-days at risk during the same period. Thus, the rate was 0.21 (95\% CI 0.19-0.22) cases of thrombotic events per 1 million vaccinated person-days. For VTE and ATE, rates were 0.075 (95\% CI $0.07-0.08)$ and 0.13 (95\% CI $0.12-0.14)$ cases per 1 million vaccinated person-days, respectively.

First and foremost, we have recorded an imbalance between VTE and arterial thrombotic ATE in mRNA vaccines: 31.8\% (381/1197) and 67.9\% (813/1197) for Comirnaty, respectively; and 24.6\% (80/325) and $77.6 \%$ (253/325) for the Moderna vaccine. Conversely, for AZD1222 we have noticed that the proportion of VTE and ATE is more evenly shared: 52.2\% (334/639) versus 48.2\% (308/639), respectively. The time frame between vaccination and ATE is the same for the three vaccines (median of 2 days), whereas we

\section{Shareable abstract (@ERSpublications)}

This study observed an imbalance between venous and arterial thrombotic events in mRNA vaccines while with AZ1222 they are evenly shared. Our analysis highlights cerebral vein thrombosis with the three vaccines. https://bit.ly/3mZqguE

Cite this article as: Smadja DM, Yue Q-Y, Chocron R, et al. Vaccination against COVID-19: insight from arterial and venous thrombosis occurrence using data from VigiBase. Eur Respir J 2021; 58: 2100956 [DOI: 10.1183/13993003.00956-2021].
\end{abstract}


TABLE 1 Clinical characteristics of patients described in the World Health Organization database of individual case safety reports, performed at the Uppsala Drug Monitoring Centre until 16 March, 2021 for the three vaccines: Comirnaty from Pfizer-BioNtech, the Moderna COVID-19 vaccine and the Oxford-AstraZeneca vaccine (AZD1222)

\begin{tabular}{|c|c|c|c|}
\hline & Comirnaty & Moderna & AZD1222 \\
\hline Total cases & 1197 & 325 & 639 \\
\hline Age years & $76(18-102)$ & $72(19-102)$ & 67 (19-99) \\
\hline \multicolumn{4}{|l|}{ Patient sex } \\
\hline Female & $708(59.1 \%)$ & $173(53.2 \%)$ & $332(52 \%)$ \\
\hline Male & $483(40.4 \%)$ & $152(46.8 \%)$ & $291(45.5 \%)$ \\
\hline Unknown & $6(0.5 \%)$ & & $16(2.5 \%)$ \\
\hline Death & $223(18.6 \%)$ & $53(16.3 \%)$ & $82(12.8 \%)$ \\
\hline Global time in days to thrombotic event & $3(0-52)$ & $2(0-63)$ & $5(0-55)$ \\
\hline Venous thrombotic events & $381(31.8 \%)$ & $80(24.6 \%)$ & $334(52.3 \%)$ \\
\hline Age years & $62(21-98)$ & $58.5(19-96)$ & $63(18-99)$ \\
\hline Time in days to thrombotic event & $4(0-50)$ & $4(0-39)$ & $6(0-55)$ \\
\hline Pulmonary embolism & $211(17.6 \%)$ & $53(16.3 \%)$ & $115(18 \%)$ \\
\hline Lower limb thrombosis & $111(9.3 \%)$ & $13(4 \%)$ & $113(17.7 \%)$ \\
\hline Cerebral venous sinus thrombosis & $3(0.3 \%)$ & $3(0.9 \%)$ & $6(0.9 \%)$ \\
\hline Cerebral venous thrombosis & $1(0.1 \%)$ & & $1(0.2 \%)$ \\
\hline Undetermined venous thrombotic event & $42(3.5 . \%)$ & $10(3.1 \%)$ & $92(14.4 \%)$ \\
\hline Others & $13(1.1 \%)$ & $1(0.3 \%)$ & $7(1.1 \%)$ \\
\hline Arterial thrombotic events & $813(67.9 \%)$ & $253(77.6 \%)$ & $308(48.2 \%)$ \\
\hline Age years & $80(18-102)$ & $75(21-102)$ & $70(21-99)$ \\
\hline Time in days to thrombotic event & $2(0-52)$ & $2(0-63)$ & $2(0-38)$ \\
\hline Stroke & $561(46.9 \%)$ & $173(53.1 \%)$ & $219(34.3 \%)$ \\
\hline Acute myocardial infarction & $238(19.9 \%)$ & $67(20.6 \%)$ & $81(12.7 \%)$ \\
\hline Stroke and myocardial infarction & $2(0.2 \%)$ & $3(0.9 \%)$ & \\
\hline Others & $12(1 \%)^{\#}$ & $10(3.1 \%)$ & $8(1.3 \%)^{4}$ \\
\hline Concomitant arterial and venous thrombotic events & $10(0.8 \%)$ & $8(2.4 \%)$ & $4(0.6 \%)$ \\
\hline Age years & $70.5(25-86)$ & $56(37-94)$ & $57.5(31-71)$ \\
\hline Time in days to thrombotic event & $3.5(0-11)$ & $2(0-22)$ & $3(1-12)$ \\
\hline Acute myocardial infarction and pulmonary embolism & $4(0.3 \%)$ & & $1(0.15 \%)$ \\
\hline Stroke and pulmonary embolism & $3(0.3 \%)$ & $6(1.85 \%)$ & $1(0.15 \%)$ \\
\hline Stroke and lower limb ischaemia & $1(0.1 \%)$ & & \\
\hline Arterio-venous fistula thrombosis & $1(0.1 \%)$ & & \\
\hline Arterial limb ischaemia and lower limb thrombosis & $1(0.1 \%)$ & & \\
\hline Stroke and lower limb thrombosis & & $1(0.31 \%)$ & \\
\hline Arterial and venous thrombosis & & $1(0.31 \%)$ & \\
\hline Pulmonary embolism and multiple thrombosis & & & $1(0.15 \%)$ \\
\hline Acute myocardial infarction and venous thrombosis & & & $1(0.15 \%)$ \\
\hline Associated thrombocytopenia and/or immunothrombosis disorders & $32(2.6 \%)$ & $8(2.4 \%)$ & $14(2.2 \%)$ \\
\hline Age years & $56(19-92)$ & $64(51-77)$ & $46.5(19-73)$ \\
\hline Time in days to event & $4.5(0-25)$ & $4(0-10)$ & $8(2-14)$ \\
\hline Thrombocytopenia associated with pulmonary embolism & $2(0.2 \%)$ & $1(0.3 \%)$ & $3(0.5 \%)$ \\
\hline Thrombocytopenia associated with acute myocardial infarction & $3(0.3 \%)$ & & \\
\hline Thrombocytopenia associated with stroke & $13(1.1 \%)$ & & $1(0.2 \%)$ \\
\hline Thrombocytopenia linked to purpura thrombotic thrombocytopenia & $7(0.6 \%)$ & & \\
\hline DIC & $2(0.2 \%)$ & & \\
\hline DIC positive lupus anticoagulant associated with visceral venous thrombosis & $1(0.1 \%)$ & & \\
\hline Positive lupus anticoagulant without thrombosis & $2(0.2 \%)$ & & \\
\hline Positive lupus anticoagulant associated with pulmonary embolism & $2(0.2 \%)$ & $1(0.3 \%)$ & \\
\hline Positive lupus anticoagulant associated with stroke & & $4(1.2 \%)$ & \\
\hline Positive lupus anticoagulant associated with myocardial infarction & & $2(0.6 \%)$ & \\
\hline Thrombocytopenia associated with splenic venous thrombosis & & & $1(0.2 \%)$ \\
\hline Thrombocytopenia with HIT-like mAb positive and multiple thrombosis & & & $1(0.2 \%)$ \\
\hline Thrombocytopenia associated with cerebral venous sinus thrombosis & & & $4(0.6 \%)$ \\
\hline Thrombocytopenia associated with cerebral venous thrombosis & & & $1(0.2 \%)$ \\
\hline DIC and pulmonary embolism & & & $1(0.2 \%)$ \\
\hline DIC and stroke & & & $2(0.3 \%)$ \\
\hline
\end{tabular}

Data are presented as $\mathrm{n}$ (percentage of cases reported) or median (range). \#: others (limb, intestinal); ": others (retinal, intestinal). DIC: disseminated intravascular coagulation; HIT: heparin-induced thrombocytopenia; mAb: antibody. 
identified a significant difference between AZD1222 (median of 6 days) and both mRNA vaccines (median of 4 days, with $\mathrm{p}=0.007$ and 0.02 , respectively, for Comirnaty and the Moderna vaccine) for VTE. Concerning ATE, the patients' profile for the three vaccines appear to be similar.

Moreover, we observed unexpected cerebral venous thrombosis (CVT) with the Moderna vaccine $(0.9 \%$ (3/325) of events reported; time to event: 2-39 days; age range: 30-37 years old; three women), with AZD1222 (1.1\% (7/639) of events reported; time to event: 2-16 days; age range: 19-59 years old; three women and four men) and with Comirnaty (0.4\% (4/1197) of events reported; time to event: $1-10$ days; age range: 30-84 years-old; four women). Three patients out of four with Comirnaty, all with Moderna vaccine and six out of seven with AZ1222 had a particular form of CVT, called cerebral sinus vein thrombosis (CVST). Five out of seven CVT cases observed after AZD1222 were associated with thrombocytopenia. Moreover, we noticed thrombocytopenia associated with thrombotic events and/or disseminated intravascular coagulation and/or antiphospholipids antibodies for all three vaccines, and one thrombocytopenia associated with HIT-positive tests after AZD1222. Since we performed the data extraction from the WHO database, several other cases of HIT have been described by two groups after AZD1222 vaccination [11, 12]. They proposed to name this phenomenon vaccine-induced immune thrombotic thrombocytopenia (VITT).

There are several limitations in our data presented here. First, for pharmacovigilance purposes, the appropriate term is reporting rate. Incidence or prevalence are not appropriate since we have no information about the precise denominator for each separate vaccine and about the extent of underreporting. Indeed, these pharmacovigilance spontaneous reports are part of the post-marketing surveillance for drugs and underreporting of adverse drug reactions is well known [13]. In a published study, the median underreporting rate across the 37 studies included was 94\% (interquartile range 82-98\%) even for serious/ severe adverse drug reactions.

Second, the best way to evaluate thrombotic events in the vaccinated population should be to match to unvaccinated controls in a 1:1 ratio according to demographic and clinical characteristics [14]. However, using adverse drug reactions reported via VigiBase does not allow us to utilise this kind of paired data. Third, unusual reporting may have occurred because of the novelty of the vaccines. Indeed, study design may modify the reporting of adverse drug reactions [15]. Open-label studies have been described to overestimate the risk of vascular adverse events by at least $50 \%$ in comparison to double blind randomised trials [15]. Pharmacovigilance spontaneous reporting is different from clinical trials, but probably close to that of open-label studies for potential unusual estimation of thrombotic events that could be influenced by the novelty of the drug, media interest and/or conflicting results in the literature.

All in all, our data represents a hypothesis-generating study suggesting that thrombotic events, including CVT, might occur in association with all three vaccines, but this hypothesis requires further investigation, including extensive clinical and biological studies. The benefit of the vaccines is a non-discussion point in COVID-19 outbreak epidemiology. However, there is an urgent need for a prospective evaluation of coagulopathy and thrombotic events to fathom rare but serious side-effects after COVID-19 vaccination, and to better characterise VITT and other thrombocytopenia associated or not with thrombotic events after the three vaccines.

David M. Smadja $\oplus^{1,2,3}$, Qun-Ying Yue $\odot^{4}$, Richard Chocron $\odot^{5}$, Olivier Sanchez $\oplus^{1,3,6}$ and Agnes Lillo-Le Louet $\mathbb{1}^{1,7}$

${ }^{1}$ Université de Paris, Innovative Therapies in Hemostasis, INSERM, Paris, France. ${ }^{2}$ Hematology Dept and Biosurgical Research Lab (Carpentier Foundation), Assistance Publique Hôpitaux de Paris-Centre (APHP-CUP), Paris, France. ${ }^{3}$ F-CRIN INNOVTE Network, Saint Etienne, France. ${ }^{4}$ Uppsala Monitoring Centre, Uppsala, Sweden. ${ }^{5}$ Emergency Dept, Université de Paris, PARCC, INSERM U970, AP-HP-Centre Université de Paris (APHP-CUP), Paris, France. ${ }^{6}$ Respiratory Medicine Dept and Biosurgical Research Lab (Carpentier Foundation), Assistance Publique Hôpitaux de Paris-Centre (APHP-CUP), Paris, France. ${ }^{7}$ Pharmacovigilance Dept, Assistance Publique Hôpitaux de Paris-Centre (APHP-CUP), Paris, France.

Corresponding author: David M. Smadja (david.smadja@aphp.fr)

Acknowledgement: This publication describes information obtained from VigiBase. VigiBase contains information from a variety of sources, and the probability that the suspected adverse effect is drug-related is not the same in all cases. This information does not represent the opinion of the Uppsala Monitoring Centre or the World Health Organization. 
Conflict of interest: Q-Y. Yue has nothing to disclose. R. Chocron reports personal fees from Aspen, outside the submitted work. O. Sanchez reports grants from Bayer, Daichi Sankyo and Portola Pharmaceuticals, personal fees from Actelion, GlaxoSmithKline, Boehringer Ingelheim and Chiesi, non-financial support from Leo Pharma, outside the submitted work. A. Lillo-Le Louet has nothing to disclose. D.M. Smadja reports personal fees from Carmat, Bayer, BMS, Aspen and Leo Pharma, non-financial support from Boehringer Ingelheim, outside the submitted work.

Support statement: D.M. Smadja's COVID team has been funded with grants from the French National Agency for Research ANR SARCODO (Fondation de France) and Mécénat Covid AP-HP. Funding information for this article has been deposited with the Crossref Funder Registry.

\section{References}

1 Philippe A, Chocron R, Gendron N, et al. Circulating Von Willebrand factor and high molecular weight multimers as markers of endothelial injury predict COVID-19 in-hospital mortality. Angiogenesis 2021; in press [https://doi.org/10.1007/s10456-020-09762-6].

2 Jevnikar M, Sanchez O, Chocron R, et al. Prevalence of pulmonary embolism in patients with COVID 19 at the time of hospital admission. Eur Respir J 2021; 58: 2100116.

3 Huertas A, Montani D, Savale L, et al. Endothelial cell dysfunction: a major player in SARS-CoV-2 infection (COVID-19)? Eur Respir J 2020; 56: 2001634.

4 Chocron R, Galand V, Cellier J, et al. Anticoagulation prior to hospitalization is a potential protective factor for COVID-19: insight from a French multicenter cohort study. J Am Heart Assoc 2021; 10: e018624.

5 Bate A, Lindquist M, Edwards IR, et al. A Bayesian neural network method for adverse drug reaction signal generation. Eur J Clin Pharmacol 1998; 54: 315-321.

6 Barker $\mathrm{Cl}$, Snape MD. Pandemic influenza A H1N1 vaccines and narcolepsy: vaccine safety surveillance in action. Lancet Infect Dis 2014; 14: 227-238.

7 Polack FP, Thomas SJ, Kitchin N, et al. Safety and efficacy of the BNT162b2 mRNA Covid-19 vaccine. N Engl J Med 2020; 383: 2603-2615.

8 Baden LR, El Sahly HM, Essink B, et al. Efficacy and safety of the mRNA-1273 SARS-CoV-2 vaccine. N Engl J Med 2021; 384: 403-416.

9 Voysey M, Costa Clemens SA, Madhi SA, et al. Single-dose administration and the influence of the timing of the booster dose on immunogenicity and efficacy of ChAdOx1 nCoV-19 (AZD1222) vaccine: a pooled analysis of four randomised trials. Lancet 2021; 397: 881-891.

10 Our World in Data. Statistics and Research: Coronavirus (COVID-19) Vaccinations. https://ourworldindata.org/ covid-vaccinations

11 Greinacher A, Thiele T, Warkentin TE, et al. Thrombotic thrombocytopenia after ChAdOx1 nCov-19 vaccination. N Engl J Med 2021; 384: 2092-2101.

12 Schultz NH, Sørvoll IH, Michelsen AE, et al. Thrombosis and thrombocytopenia after ChAdOx1 nCoV-19 vaccination. N Engl J Med 2021; 384: 2124-2130.

13 Hazell L, Shakir SA. Under-reporting of adverse drug reactions: a systematic review. Drug Saf 2006; 29: 385-396.

14 Dagan N, Barda N, Kepten E, et al. BNT162b2 mRNA Covid-19 vaccine in a nationwide mass vaccination setting. N Engl J Med 2021; 384: 1412-1423.

15 Trone JC, Ollier E, Chapelle C, et al. Statistical controversies in clinical research: limitations of open-label studies assessing antiangiogenic therapies with regard to evaluation of vascular adverse drug events-a meta-analysis. Ann Oncol 2018; 29: 803-811. 